\title{
Novel isosorbide di-ester compounds as inhibitors of acetylcholinesterase
}

\author{
Ciaran G. Carolan ${ }^{\mathrm{a}}$, Joanne M. Gaynor ${ }^{\mathrm{b}}$, Gerry P. Dillon ${ }^{\mathrm{a}}$, Denise Khan ${ }^{\mathrm{a}}$, Sheila A. Ryder ${ }^{\mathrm{a}}$, Sean Reidy ${ }^{\mathrm{b}}$, John F. Gilmer ${ }^{\mathrm{a}}$ \\ ${ }^{a}$ School of Pharmacy and Pharmaceutical Sciences, Trinity College Dublin, Dublin 2, Ireland \\ ${ }^{\mathrm{b}}$ School of Science, Athlone Institute of Technology, Athlone, Ireland \\ E-mail: carolanc@tcd.ie (C.G. Carolan)
}

NOTICE: this is the author's version of a work that was accepted for publication in Chemico-Biological Interactions following peer review. Changes resulting from the publishing process, such as peer review, editing, corrections, structural formatting, and other quality control mechanisms may not be reflected in this document. Changes may have been made to this work since it was submitted for publication. A definitive version was subsequently published in Chemico-Biological Interactions [Vol. 175, Issues 1-3 (Sep 2008)], DOI: 10.1016/j.cbi.2008.05.013.

Direct links to the definitive publisher-authenticated version [Carolan CG, Gaynor JM, Dillon GP, Khan D, Ryder SA, Reidy S, Gilmer JF. Novel isosorbide di-ester compounds as inhibitors of acetylcholinesterase. Chem Biol Interact. 2008 Sep 25; 175(1-3):293-7]:

http://www.sciencedirect.com/science/article/pii/S0009279708002597

http://dx.doi.org/10.1016/j.cbi.2008.05.013

\begin{abstract}
We report herein that a variety of isosorbide di-esters, previously reported to be novel substrates for butyrylcholinesterase (BuChE, EC 3.1.1.8), are in fact inhibitors of the homologous enzyme acetylcholinesterase (AChE), with $I_{50}$ values in the micromolar range. In vitro studies show that they are mixed inhibitors of the enzyme, and thus the ternary enzymeinhibitor-substrate complex can form in acetylcholinesterase. This is rationalised by molecular modelling which shows that the compounds bind in the mid-gorge area. In this position, simultaneous substrate binding might be possible, but the hydrolysis of this substrate is prevented. The di-esters dock within the butyrylcholinesterase gorge in a very different manner, with the ester sidechain at the 5-position occupying the acyl pocket at residues Leu286 and Val288, and the 2-ester binding to Trp82. The carbonyl group of the 2- ester is susceptible to nucleophilic attack by Ser198 of the catalytic triad. The larger residues of the acyl pocket in acetylcholinesterase prevent binding in this manner. The results complement each other and explain the differing behaviours of the esters in the cholinesterase enzymes. These findings may prove very significant for future work.
\end{abstract}

Keywords Acetylcholinesterase $\bullet$ Butyrylcholinesterase $\bullet$ Isosorbide esters $\bullet$ Inhibitor $\bullet$ Docking

\section{Introduction}

Acetylcholinesterase (AChE, EC 3.1.1.7) is present throughout the central and peripheral nervous systems of mammals, where it catalyses the hydrolysis of the endogenous ester neurotransmitter acetylcholine (ACh), allowing the termination of ACh receptor-mediated ion gating at nerve-nerve and neuromuscular junctions. ACh is also hydrolysed by the enzyme butyrylcholinesterase (BuChE, EC 3.1.1.8), which although present in the human body, has no known physiological role. The enzymes are very similar, possessing 53\% sequence homology and both having their active sites at the base of an enzymatic gorge of depth $20 \AA$.

This group previously reported that certain isosorbide esters (Fig. 1) are hydrolysed extremely rapidly by BuChE [1]. Here we report the results of studies on the interactions of these isosorbide esters with AChE, which show that these compounds are actually reversible inhibitors of AChE, as opposed to substrates for the enzyme as was the case in BuChE. It is puzzling in the first instance that ester compounds might strongly bind the enzyme but not be hydrolysed, and this is even truer when the homologous BuChE rapidly hydrolyses these same compounds. Through kinetic analyses and in silico modelling,we sought to explain this unexpected finding.

\section{Experimental methods}

\subsection{Chemicals}

Acetylthiocholine iodide (ATCI), 5,5'-dithiobis-(2-nitrobenzoic acid) (DTNB) and acetylcholinesterase were purchased from Sigma-Aldrich, Ireland. The acetylcholinesterase used was the Type V-S protein from Electrophorus electricus (electric eel), purchased as a lyophilised powder. It was solubilized in $0.02 \mathrm{M}$ phosphate buffer $\mathrm{pH} 7.0$, stabilised by addition of $1 \mathrm{mg} / \mathrm{ml}$ bovine serum albumin (BSA), and refrigerated until use. 


\subsection{Synthesis of isosorbide-based esters}

The di-esters were prepared from isosorbide using the appropriate acid chloride in the presence of a tertiary base. They were obtained as crystalline materials following chromatography and characterised by both NMR and MS.

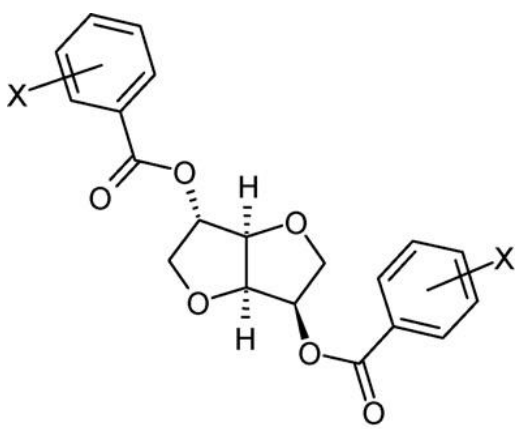

Fig. 1. The structure of the di-benzoate compounds tested. $X$ represents either a chlorine or bromine substituent on the aromatic rings.

\subsection{Inhibition assays}

Studies using RP-HPLC showed that the di-esters were not hydrolysed when they were incubated with E. electricus AChE; instead, the esters were inhibitors. Inhibition of the E. electricus AChE by the di-esters was assayed using the method of Ellman et al. [2], modified to allow the assay to be run in 96-well plate format.

E. electricus AChE and 5,5'-dithiobis-(2-nitrobenzoic acid) (DTNB) were incubated in phosphate buffer solution pH 8.0 at $37^{\circ} \mathrm{C}$ at final concentrations of $0.05 \mathrm{U} / \mathrm{ml}$ and $0.3 \mathrm{mM}$, respectively. Inhibitors were incubated in this enzymatic solution for $30 \mathrm{~min}$ at concentrations between $100 \mathrm{nM}$ and $100 \mu \mathrm{M}$, prior to addition of the substrate acetylthiocholine iodide; concentrations of ATCl ranged between $100 \mu \mathrm{M}$ and $5 \mathrm{mM}$ in the final solution. The absorbance of the resulting solutions was monitored at $405 \mathrm{~nm}$. In each resulting plot, data points were obtained on the basis of readings from at least four replicate wells.

Plots of $1 / v$ against $[I]$ and $s / v$ against $[I]$ were prepared to examine the nature of the inhibition by each test compound. $(v$, reaction rate; $s$, substrate concentration; and $[I]$, inhibitor concentration). Values for $K_{i}$ and $K_{i}^{\prime}$ were calculated by plotting $K_{m} / V_{\max }$ and $1 / V_{\max }$ against [I], where $K_{m}$ and $V_{\max }$ were calculated by fitting data to the Michaelis-Menten equation using non-linear regression. In each case, a straight line was obtained, and the intercept on the $[I]$ axis gave $-K_{i}$ when $K_{m} / V_{\max }$ was plotted, and $-K_{i}^{\prime}$ when $1 / V_{\max }$ was plotted [3]. All data manipulations and graphing calculations were carried out using GraphPad Prism version 4.03 (GraphPad Software).

\subsection{Molecular modelling}

The binding of the di-ester compounds to both BuChE and AChE was examined to see whether differential binding to each enzyme could explain the different behaviour of the di-esters in the enzymes. Isosorbide di-(4-chlorobenzoate) was docked to the active sites of both hBuChE and E. electricus AChE. Isosorbide di-(4-bromobenzoate) was also docked to AChE.

Docking calculations were carried out using AutoDock version 4.0, with the Lamarckian genetic algorithm (LGA) [4]. The inhibitor was built using the builder function of MOE (Chemical Computing Group, Montreal, Canada) and minimized with MOPAC 7 (PM3 method) interfaced to MOE.

The crystal structure of hBuChE in complex with a choline molecule [5] (PDB code 1POM) was used as a basis for modelling the interaction with BuChE. The missing sidechain of residue Gln486 was added in Swiss-PDB Viewer version 3.7, and the missing residues 1-3, 378-379 and 455 added using the 'Add residue' command in the same program. These added residues were minimized within $\mathrm{MOE}$, with the rest of the macromolecule held fixed. Of the two conformations of the catalytic serine observed in the crystal structure noted above, the one where it makes the anticipated hydrogen bond with His438 was used. His 438 was protonated at the $\delta$ position. The substrates and the enzyme were further prepared for the docking calculation using AutoDockTools 1.4.5. Rotation was allowed for all usual bonds in the ligand, but the macromolecule was held rigid. The 3D affinity grid box was designed to include the full active site gorge of human BChE. The number of grid points in the $x-, y-, z$-axes was 64,64 , and 40 with grid points separated by $0.375 \AA$. One hundred docking runs were completed, with $25,000,000$ energy evaluations allowed as a maximum in each run. The lowest energy result was taken as the correctly docked pose.

An identical approach was undertaken to dock the same ligand to AChE. Since high-resolution crystal structures of $E$. electricus AChE were not available, the 1.8 A resolution structure of the homologous Torpedo californica AChE was used for modelling purposes [6] (PDB Code 1EA5). The catalytic domains of the two proteins present 68\% identity [7], while the residues of the active site gorge are even more closely matched. However, residue Phe330 in the active site gorge of Torpedo AChE is replaced by a tyrosine residue, while lle439 - also close to the gorge - is replaced by a proline in the protein from the electric eel. In order to take into account these and other differences, a homology model was built for $E$. electricus AChE using the T. californica structure as a template. 
The homology-model application of MOE was used for this purpose. In building the initial geometry, all coordinates were copied for residues whose identity was conserved in both proteins, while backbone coordinates were copied for others. Loops and missing sidechains were added from generic libraries within MOE to prepare 10 different models. After addition of hydrogen atoms and coarse minimization, the highest scoring protein based on electrostatic solvation energy, calculated using a Generalized Born/Volume Integral (GB/VI) methodology, was taken forward for final model building. It was minimised to a root mean square gradient of 0.01 . This final model was checked for appropriate geometry before use.

The di-benzoate compounds were docked within the active site of the modelled protein. The number of grid points in the $x$-, $y$ - and $z$-axes was 54, 58 and 56 in this case, but all other steps were as described for BuChE modelling.

\section{Results}

All isosorbide di-ester compounds tested were inhibitors of $\mathrm{AChE}$, with $K_{i}$ values in the $\mu \mathrm{M}$ range, as shown in Table 1 . The most potent inhibitors were isosorbide di-(3-chlorobenzoate) and isosorbide di-(4-chlorobenzoate), with $K_{i}$ values of 7.79 and $6.60 \mu \mathrm{M}$, respectively. The di-(4-bromobenzoate) was significantly less potent.

Table 1. $K_{i}$ and $K_{i}^{\prime}$ values for selected isosorbide di-esters, calculated as described in the text.

\begin{tabular}{lcc}
\hline Compound name & $K_{i}(\mu \mathrm{M})$ & $K_{i}^{\prime}(\mu \mathrm{M})$ \\
\hline Isosorbide di-(2-chlorobenzoate) & 43.31 & 1286 \\
Isosorbide di-(3-chlorobenzoate) & 7.79 & 50.4 \\
Isosorbide di-(4-chlorobenzoate) & 6.60 & 92.6 \\
Isosorbide di-(4-bromobenzoate) & 104.90 & 4361 \\
\hline
\end{tabular}

We subsequently sought to characterise the inhibition of AChE by the isosorbide esters. Plots of $1 / v$ against $[I]$ and $s / v$ against $[I]$ together were used to investigate the mode of linear inhibition, as described by Cornish-Bowden [3]. These plots were prepared from data obtained at different substrate and inhibitor concentrations in E. electricus AChE. The lines intersected just above the [I]-axis in the Dixon plot and just below the axis in the plot of $s / v$ against $[I]$, indicating mixed inhibition. This type of inhibition would most likely arise in our system if the ternary EIS complex can form but cannot break down to products. We could characterise the $K_{i}$ value as the dissociation constant of El and $K_{i}^{\prime}$ as the inhibitor dissociation constant of the EIS complex.

Models of isosorbide di-(4-chlorobenzoate) in BuChE and AChE indicated why the isosorbide esters might be substrates in BuChE but inhibitors of AChE. In BuChE, numerous docked poses scored highly, indicating that there are many stable structures formed upon binding, and between which the docked molecule could cycle within the active site gorge of the enzyme. The highest scoring pose, however, sees the di-benzoate lining the base of the gorge, as in Fig. 2 . The ester group at the 5-position occupies the acyl pocket between residues Leu286 and Val288, while the 2-ester function is orientated in the direction of Trp82, which normally binds the cationic head of the choline substrate through cation- $\pi$ interactions. The carbonyl carbon at the 2-position is $4.6 \AA$ from the nucleophilic O- $\gamma$ of Ser198. More importantly, the carbonyl oxygen is directed away from the catalytic serine and towards the oxyanion hole residues Gly116, Gly117 and Ala199. The residue is placed almost optimally for nucleophilic attack of Ser198 at the carbonyl carbon of the 2-ester, which will result in substrate hydrolysis at this position. The ester at the 5-position was not hydrolysed appreciably when tested, and this can also be explained using this same model. The carbonyl group is inappropriately placed for attack. Furthermore, this ester group is endo to the $\mathrm{V}$-shaped isosorbide ring, meaning that the approach of the catalytic serine is sterically hindered.

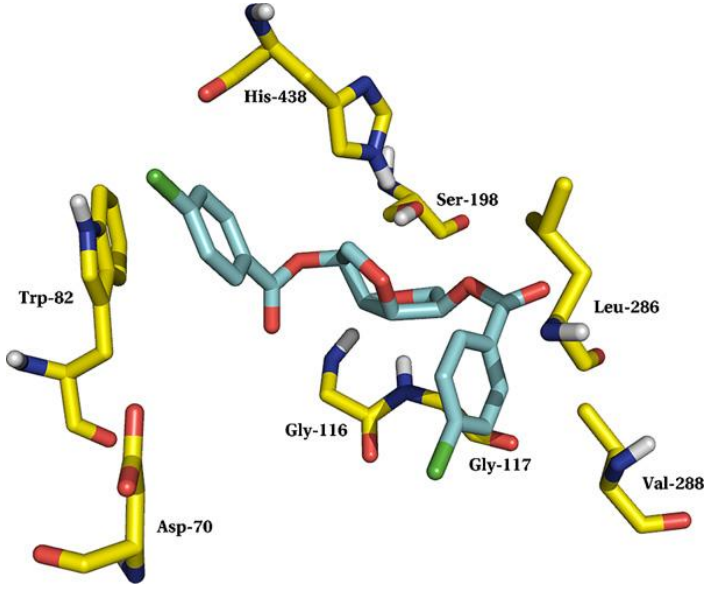

Fig. 2. Isosorbide di-(4-chlorobenzoate) docked to hBuChE.

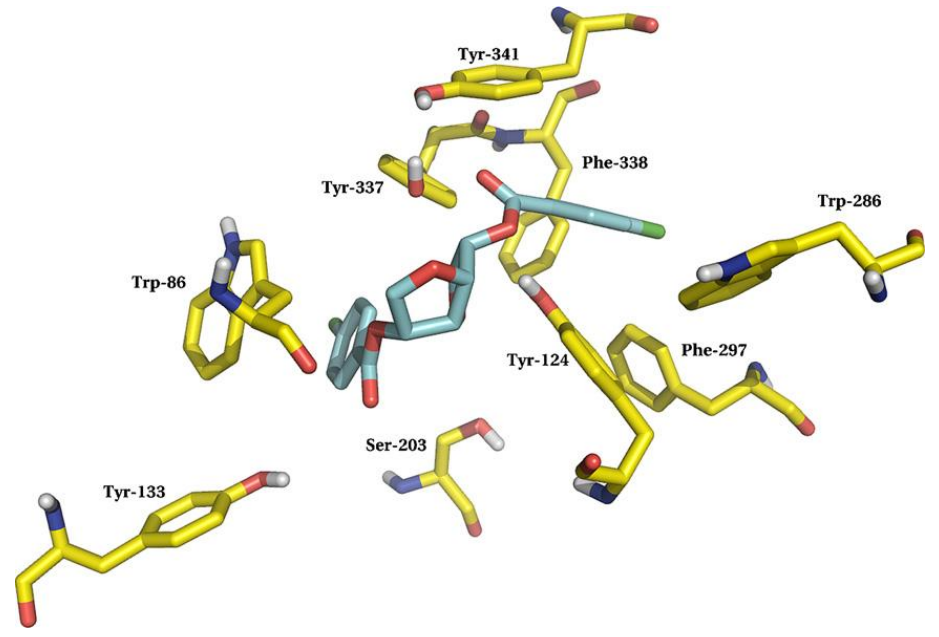

Fig. 3. Isosorbide di-(4-chlorobenzoate) docked to AChE. 
The active site gorge in AChE is more cramped, and this means that a wholly different docking pose is adopted. The acyl pocket is occluded by the presence of residues Phe295 and Phe297 (equivalent to residues Phe288 and Phe290 in T. californica enzyme), among others, so that the molecule cannot fit within the gorge in the same manner as in BuChE. The di-ester sits higher up the gorge, as shown in Fig. 3, with the more stable 5-ester group binding to Trp86 and closest to the catalytic triad residues, notwithstanding the fact that it is poorly placed for hydrolysis. The carbonyl carbon of the labile 2benzoate is $10.7 \AA$ from $0-\gamma$ of Ser198, and is bound in an aromatic pocket formed by residues Phe297, Phe338, Tyr337, Tyr341 and Trp286. Thus, the docking is well stabilized, but further descent within the gorge in order to engage the catalytic machinery is not possible, and hence this molecule acts as an inhibitor of AChE.

Isosorbide di-(4-bromobenzoate) was also docked within the active site of E. electricus AChE as described above. It docked precisely as described for the chlorobenzoate, and with an almost identical docking score. It was thus puzzling that it was a significantly less potent inhibitor in the in vitro tests. We suggest that reduced binding might arise because of the reduced electron density redistribution that occurs in the presence of bromine compared to chlorine substituents; this has been used to explain the improved binding of chlorotacrine to AChE compared to tacrine itself [8]. Otherwise, the increased steric bulk of bromine substituents may mean that the optimal binding pose described above is not as readily achieved.

\section{Discussion}

The results of the in vitro analyses and molecular modelling work described complement each other and reveal much about the nature of the binding of isosorbide di-esters to the cholinesterase enzymes, and the nature of the cholinesterase enzymes themselves. The in vitro work showed all di-esters to be mixed inhibitors of moderate potency in AChE, as opposed to extremely good substrates for BuChE, with a similar affinity for the enzyme to the prototypical substrate butyrylcholine [9]. Mixed inhibitors form complexes with their target enzymes so that typically, the ternary EIS complex can be formed, as well as EI and ES complexes. This ternary complex does not break down to form catalytic products. Thus, while the inhibitor is not strictly competitive with the substrate, and the substrate can still bind to the enzyme in the presence of the inhibitor, its presence prevents substrate turnover due to interference with the enzyme's function or with the appropriate binding of the substrate. The molecular docking of isosorbide di-(4-chlorobenzoate) to AChE reveals how this might be better understood. The di-ester cannot bind directly at the active site - if it could, it would be hydrolysed as is the case in BuChE. Instead, it binds within the gorge as depicted. In this orientation, it can be envisaged that the substrate should still be able to enter the active site; however, its binding in an appropriate manner is blocked by the presence of the inhibitor, and hence no substrate turnover is observed in this scenario.

The results of these studies have much significance for future work. These studies have shown that molecules based on the isosorbide template can bind both $\mathrm{AChE}$ and $\mathrm{BChE}$, but very different outcomes can arise from their binding in either case. Subsequent studies have begun to examine the binding of isosorbide 2-carbamates to the enzymes. Initial results have shown these compounds to be selective for BuChE in most cases, with strong pseudo-irreversible inhibition observed [10]. In AChE, though inhibition is still apparent, it is only moderate. This might be anticipated from these studies of reversible inhibitors, since the carbamylation reaction should take place more quickly in BuChE if binding occurs in the same way as for the di-esters. Thus, the isosorbide template provides promise for future compounds that might have differential effects in BuChE and AChE.

\section{Conclusions}

A number of in vitro studies have shown that isosorbide di-esters are mixed inhibitors of AChE as opposed to substrates for BuChE as reported previously. Molecular modelling work shows the different binding modes in each enzyme that give rise to these different results. The isosorbide template might be useful for subsequent work where compounds with such contrasting activities in the cholinesterase enzymes are desirable.

\section{References}

[1] J.F. Gilmer,M.N. Lally, P. Gardiner,G. Dillon, J.M.Gaynor, S.Reidy, Novel isosorbide-based substrates for human butyrylcholinesterase, Chem. Biol. Interact. 157-158 (2005) 317-319. PMID: 16266694. DOI: 10.1016/j.cbi.2005.10.095.

[2] G.L. Ellman, K.D. Courtney, V. Andres Jr., R.M. Featherstone, A new and rapid colorimetric determination of acetylcholinesterase activity, Biochem. Pharmacol. 7 (1961) 88-95. PMID: 13726518. DOI: 10.1016/0006-2952(61)90145-9.

[3] A. Cornish-Bowden, Principles of Enzyme Kinetics, first ed., Butterworths, London, 1976.

[4] G.M. Morris, D.S. Goodsell, R.S. Halliday, R.Huey,W.E. Hart, R.K. Belew, A.J. Olson, Automated docking using a Lamarckian genetic algorithm and an empirical binding free energy function, J. Comput. Chem. 19 (1998) 1639-1662. DOI: 10.1002/(SICI)1096987X(19981115)19:14<1639::AID-JCC10>3.0.CO;2-B.

[5] Y. Nicolet, O. Lockridge, P. Masson, J.C. Fontecilla-Camps, F. Nachon, Crystal structure of human butyrylcholinesterase and of its complexes with substrate and products, J. Biol. Chem. 278 (2003) 41141-41147. PMID: 12869558. DOI: 10.1074/jbc.M210241200.

[6] Harel M, Weik M, Silman I, Sussman JL, Native acetylcholinesterase (E.C. 3.1.1.7) from Torpedo californica at $1.8 \AA$ resolution, in press (structure available online at http://www.pdb.org). 
[7] S. Simon, J. Massoulié, Cloning and expression of acetylcholinesterase from Electrophorus. Splicing pattern of the 3' exons in vivo and in transfected mammalian cells, J. Biol. Chem. 272 (1997) 33045-33055. PMID: 9407087. DOI: 10.1074/jbc.272.52.33045.

[8] S.T. Wlodek, J. Antosiewicz, J.A. McCammon, T.P. Straatsma, M.K. Gilson, J.M. Briggs, C. Humblet, J.L. Sussman, Binding of tacrine and 6-chlorotacrine by acetylcholinesterase, Biopolymers 38 (1996) 109-117. PMID: 8679940.

[9] W. Xie, C.V. Altamirano, C.F. Bartels, R.J. Speirs, J.R. Cashman, O. Lockridge, An improved cocaine hydrolase: the A328Y mutant of human butyrylcholinesterase is 4-fold more efficient, Mol. Pharmacol. 55 (1999) 83-91. PMID: 9882701.

[10] J.M. Gaynor, G.P. Dillon, S. Reidy, J.F. Gilmer, Synthesis and structure activity relationships (SAR) of a new class of potent and selective butyrylcholinesterase inhibitors, Chem. Biol. Interact. 157-158 (2005) 380-381. PMID: 16429505. DOI: 10.1016/j.cbi.2005.10.063. 